\title{
Buffering Management Scheme for Multimedia Synchronization in Mobile Information System
}

\author{
Gi-Sung Lee ${ }^{1}$, Jeung-gyu Jee ${ }^{2}$, and Sok-Pal Cho ${ }^{3}$ \\ 1 Dept. of Computer Science, Howon Univ., Korea \\ ygslee@sunny.howon.ac.kr \\ 2 Korea Research Foundation, Korea \\ 3 Dept. of C\&C . Eng. Sungkyul Univ., Korea
}

\begin{abstract}
In this paper we propose on buffering management scheme to address the problem how to maintain a smoothing stream presentation in multimedia mobile environments. For this case, a feedback scheme is used for sustaining a continuous presentation of multimedia stream and a presentation control scheme for maintaining a normal level of buffer. The proposed scheme shows not only a seamless play-out but also the higher play-out rate and the lower loss rate.
\end{abstract}

\section{Introduction}

The explosive growth of Internet access in parallel with the technological advances in mobile communications has motivated multimedia applications in mobile networks. For this reason, user's need for diverse multimedia services can't be admitted with the previous client/server based service structure. This limitation can be overcome by extending the concept of service providing systems to mobile systems connected with the mobile networks. Particularly, the interest of a distributed store technique for multimedia services is increasing in mobile information environments. It is different to play out multimedia data in a Mobile Host(MH) in mobile environments because high data loss rate and delay, and low bandwidth. For these reason, buffer is used in the distributed multimedia systems. The Base Station(BS) receives many sub-frames from multimedia servers. However the unexpected delay and the increase in traffics result in the decrease in the possibility of playing out sub-frames, in the case where the playout time in the BS is faster then the arrival time from the multimedia server. The buffer at the BS provide temporary storage for multimedia data, in order to smooth potential delay and jitter introduced by the wired network and facilitate synchronization between the multimedia servers and the BS. Once the play-out starts, data is delivered with little delay variation. Among the previous buffering schemes for multimedia synchronization, a forward feedback scheme has a problem that it may not control the number of sub-frames in case of the underflow and the overflow occurrence. Due to this reason, the buffer level is always controlled with the same level, and this results in the media loss. In this paper we propose a smoothing buffering management scheme for sustaining a continuous 
presentation for multimedia stream, and a presentation control scheme for maintaining a stately level of the buffer. As a result of the proposed methods, the safe state of buffers is continuously maintained because the filtering function and the network state variables are reflected in the value of feedback, and the play-out time is adaptively modified. $\mathrm{MH}$ is also absolved from his responsibility for synchronization because the synchronization for multimedia data is performed only in BS.

\section{Related Work}

In [4 [7, a start-up time before transmitting streams is defined by sending a roundtrip packet in evaluation phase, and the skew problem between the buffer size and media size is solved by readjusting the number of servers with the maximum jitter and the first transmission time in other servers. This results in the increase in play-out rate by maintaining the safe state of buffer. However, it takes much time to move into the safe state from the underflow or the overflow because the network state is reflected in the value of filtering function needed to maintain the safe state. It is also hard to sustain the safe state because this method doesn't consider the play-out time. In[5], a BS serves as an interface between the wired and the wireless networks, and buffering at the BS for interfacing with the wired networks is considered to smooth inter-packet jitter delay. This method attempts only to apply synchronization to channel allocation. The buffer management and the feedback scheme in which the received multimedia units are registered to the buffer at a $\mathrm{MH}$, and their play-out time is controlled based on the middle of the buffer size, is studied in [2] [3]. However this method can not support the required QoS level by changing a play-out time even though the state of wireless networks is normal. In 1, a synchronization scheme for wireless clients and distributed multimedia systems using a quasi-sink to control synchronization was proposed. This method defines efficiently the role of system elements such as multimedia server, BS, and MH. However, the synchronization for multimedia data is performed only in the start point of transmission. That is, there is no policy for play-out in this method.

\section{Buffering Management Scheme for Multimedia Synchronization}

\subsection{System Model in Mobile Networks}

Fig.1 shows the system model in mobile networks. The overall system configuration consists of three parts: multimedia servers $\mathrm{k}$, BSs m and MHs n. A BS should communicate with MHs $\mathrm{n}$, and a MH should access multimedia servers over wired networks through a BS. This system allows the BSs to manage the buffers and the transmission time using the arrival time and delay jitter from the multimedia servers. This provides a solution to the problems of the $\mathrm{MH}$ with small memory and the BS with the limited resources than the sites in the wired 
networks. In this system, a movie that is divided into objects $\mathrm{i}$ is separately registered to servers $\mathrm{k}$. This system has the minimization of start up time and the decrease of the network traffic and the buffer size. A multimedia data can be divided into smaller Sprit Streams with logical time using a sub-frame stripping technique, and is separately registered to each server. The smaller stream is referred as a sub-frame. A message manager in a multimedia server skips and transmits sub-frames using the offset of a control function from a feedback manager of a BS. The BS registers the arrival time of sub-frames from each server, and gets the transmission start times from multimedia servers through dummy packets. A communication manager in a multimedia server classifies feedback packets and messages from a BS, and reports this information. A communication manager in a BS sorts the ordering of the sub-frames transmitted from a multimedia server.

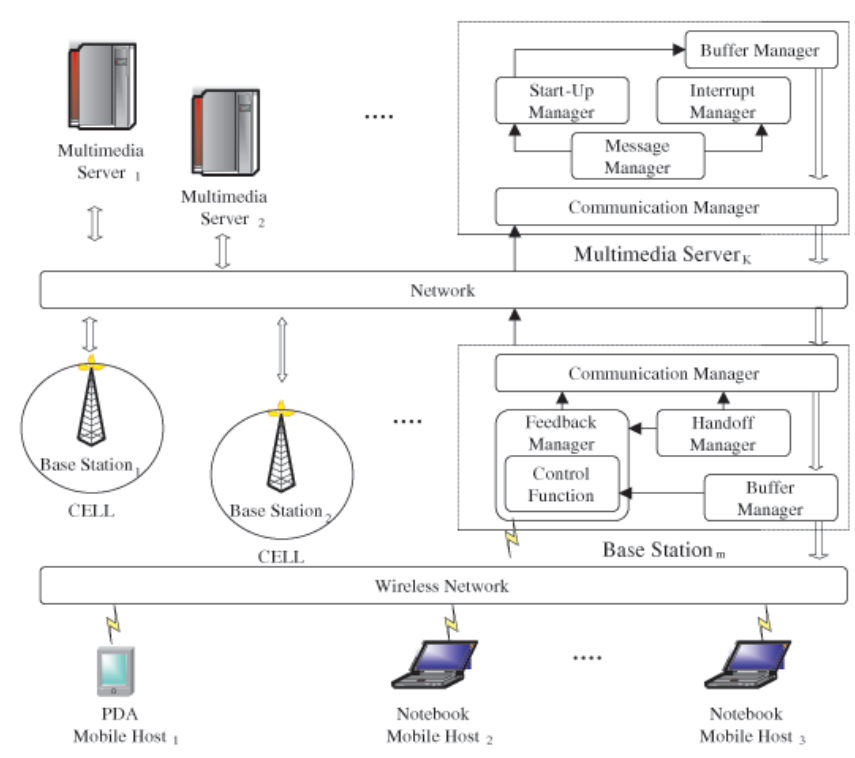

Fig. 1. System model in mobile networks

\subsection{Buffering Management Structure}

As shown in Fig. 2, a buffer manager in a BS controls the state of buffers. The buffer state can be classified into five levels: normal level, upper threshold level, upper control level, lower threshold level and lower control level.

1. normal level

The state of the buffer is normal level. In this case, the buffer manager receives continuously media data. It also checks sub-frame numbers and the jitter scope, and registers the checked information. 
2. upper threshold level and upper control level

The possibility of suffering from the buffer overflow gets higher if the buffer reaches the upper threshold level, and the buffer overflow is originated if the buffer reaches the upper control level. If the state of the buffer is the upper threshold level, the buffer manager informs a $\mathrm{MH}$ of the sub-frames and the play-out time obtained from feedback policy of a feedback manager.

3. lower threshold level and lower control level

The possibility of suffering from the buffer underflow gets higher if the buffer reaches the lower threshold level, and the buffer underflow is originated if the buffer reaches the lower control level. If the state of the buffer moves to the upper threshold level, the manager of the buffer level informs to a $\mathrm{MH}$ of a new play-out time obtained from a feedback policy of a feedback manager.

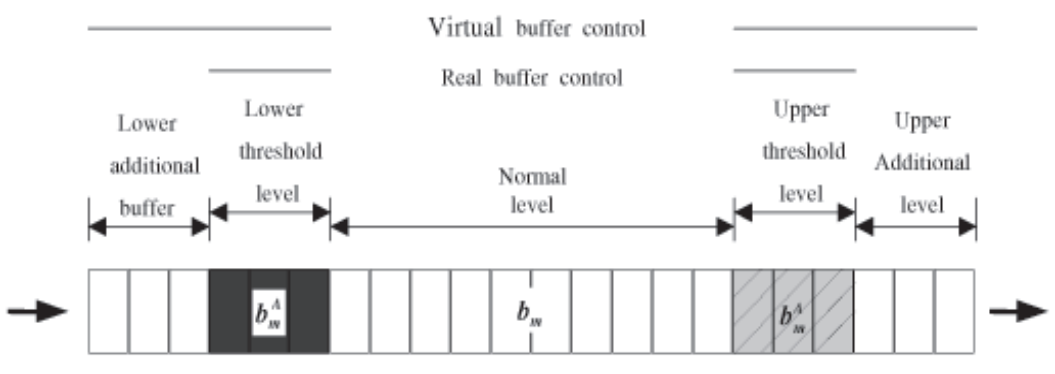

Fig. 2. Buffering State in a BS

\subsection{Buffering Management Algorithm}

We describe an algorithm in which the arrival rate and the play-out rate are adaptively adjusted to maintain the safe state in the buffer in a BS. The proposed algorithm is the following thing.

Procedure Buffering Management Algorithm

Procedure Start-up Time

Begin

$B S_{\text {time }}=$ Current BS Time;

For $i=0,1<k, i++$ do

$\operatorname{Send}\left(S_{i}\right.$, DummyPacket $\left._{i}\right) ; \quad / * S_{i}$ is each multimedia server. This function sends dummypackets to each server*/ 
Receive $\left(S_{i}, D_{k}^{i}\right.$, Dummypacket $\left.{ }_{i}\right) ;{ }^{*} D_{k}^{i}$ is $i$ th delay time of a server $K$.

This function computes the round trip time of each server $* /$ Start- $\mathrm{Up}_{i}=\operatorname{Max} D_{k}^{i}-D_{K}^{i} ; / * \operatorname{Max} D_{k}^{i}$ is the maximum delay time,

Start- $\mathrm{Up}_{i}$ denotes the transmission start time*/

Set Start-Up Time for Server $S_{k} / *$ Set the thransmission srart time for each multimedia server $* /$

End For

End /*Procedure Start-Up Time*/

Procedure Feedback /*Buffer Point at Normal Lever in BS*/

Begin

$q_{t, m}=$ current buffer_point;

$C\left(q_{t, m}\right)=\alpha \cdot \bar{b}_{t-1, m}+(1-\alpha) \cdot q_{t, m} ; / * \bar{b}_{t-1, m}$ is smoothed buffer, $\alpha$ is smoothing factor, $q_{t, m}$ is the buffer level, a filtering function*/

If $D T_{k}^{i}==$ First Variance Then $\quad / *$ The extent of the first variance in the average arrival time $* /$

$O_{t, m}=q_{t, m}-\bar{b}_{t, m}-N_{p} ; / * N_{p}$ is Mobile Network State and the value is $1 .^{*} /$

Else If $D T_{k}^{i}==$ Second Variance Then $/{ }^{*}$ The extent of the second variance in the average arrival time */

$O_{t, m}=q_{t, m}-\bar{b}_{t, m}-N_{p}$

${ }^{*} N_{p}$ is Mobile Network State, and the value is $2 * /$

End If

End

Procedure Nomal_Level

/*Buffer Point at Normal Level in BS */

Begin

$\rho_{i}=125$;

/* A expected play-out time $* /$ 
If buffer_point $==$ Normal level Then

$\tau_{t}=\rho_{i}$

$/ * \tau_{t}$ is real play-out time $* /$

End If

End

Procedure Upper_level

/* Buffer Point at Upper Level in a BS */

Begin

$B L_{\text {allsize }}=B L_{\text {allsize }}+1 ; / * B L_{\text {allsize }}$ is the number of the buffers corresponding to thethreshold level*/

$B L_{\text {psize }} ; \quad \quad / * B L_{\text {psize }}$ is the current number at upper threshold level $* /$

$\omega=0 ; / *$ A weight value $* / \rho_{i}=125$;

If buffer_point $==$ Upper level Then

Begin

$\omega=B L_{\text {allsize }} / B L_{\text {psize }} ; \tau_{t}=\rho_{i}-\left(\rho_{i} \times \omega\right)$

End If

End

Procedure Lower_level $\quad$ /* Buffer Point at Lower Level in a BS */

Begin

$B L_{\text {allsize }} ;$ BLpsize $; \omega=0 ; \rho_{i}=125 ; \lambda=10$

${ }^{*} \lambda$ is the maximum delay jitter $* /$

If buffer_point $==$ Lower level Then

Begin

$$
\omega=B L_{\text {allsize }}-B L_{\text {psize }} ; \omega=\omega / B L_{\text {allsize }} ; \tau_{t}=\rho_{i}+\lambda \times \omega ;
$$

End If 
End

Begin /* Start Main Procedure */

Call Start-Up Time; $\Delta=D T_{k}^{\max }-D T_{k}^{\min }$; /* $\Delta$ is jitter $* /$

If buffer_point $==$ Normal level Then

call normal level;

Else If buffer-point $==$ Upper level Then

Begin

If $D T_{k}^{i}<\Delta$ Then $\quad /^{*} D T_{k}^{i}$ is the sub-frame's delay time */

Begin

Feedback_value $=$ Call Feedback;

Send $\left(S_{k}\right.$, Feedback_value, $\left.D T_{k}^{i}\right)$

End

Else

Continue;

Call Upper_Level;

End

Else If buffer_point $==$ Lower level Then

Begin

Feedback_value $=$ Call feedback;

Send $\left(S_{k}\right.$, Feedback_value, $\left.D T_{k}^{i}\right)$;

End

Else 
Continue;

Call Lower_Level;

End

End If

End

End

/* Buffering Management Algorithm */

\section{Performance Evaluation}

We describe a simulation model that developed to evaluate the performance of our buffering management scheme. In our simulation environments, the interface and algorithm has been implemented by Java development kit JDK 1.3 and the result has been stored as a simulation.mdb in MDB. 1Kbyte of audio data is encoded by PCM, and resolution of video frame is 120X120. Twenty-four frames are used for encoding. It is assumed that wired networks are used for communication between BSs and multimedia servers, wireless networks for the communication between MHs and BSs. To carry out a proper work for each packet, the information used in actual simulation was calculated with Poisson distribution and the network delay time was applied equally to two types of the media. The number of frames is 1,000 , and the maximum delay jitter time is $600 \mathrm{~ms}$. The play-out rate and loss rate using the presentation time with the feedback policy and play-out control policy to keep the buffer level at a normal state is compared to the previous methods. Fig. 3 shows the buffer water-marker wherein The $\mathrm{x}$-axis indicates the number of frames and the $\mathrm{y}$-axis the buffer level. It indicates that the frame number in a buffer is delayed from 200 to 350 and thus the buffer is getting out of a normal state because the arrival time of frames is delayed. Fig. 3 also shows the state in which the buffer water-marker changes to the normal state by the feedback policy with the feedback management. It also shows that underflow is reached later than that by the previous policy. It is known that it is faster to progress to a normal state in variance 1 than that by a previous method. Also, underflow reached later variance 2 better than variance 1. The overflow occurs between 800 and 960 frames as shown in Fig. 3 We can see buffer water-marking of variance 1 and 2 is stable then that of the previous scheme. It also shows that the previous schemes don't control the overflow between 200 and 360 frames, while the proposed scheme prevents the underflow between 200 and 300 frames. The overflow state can be controlled between 800 and 960. Fig. 4 shows results of the play-out policy with buffer water-marker. It indicates that the frames between 200 and 350, and between 720 and 820 are waited and skipped, respectively. The play-out time is changed 
between 200 and 350, and also the overflow gets to be reduced by the decrease in play-out time from 750 to 960 .

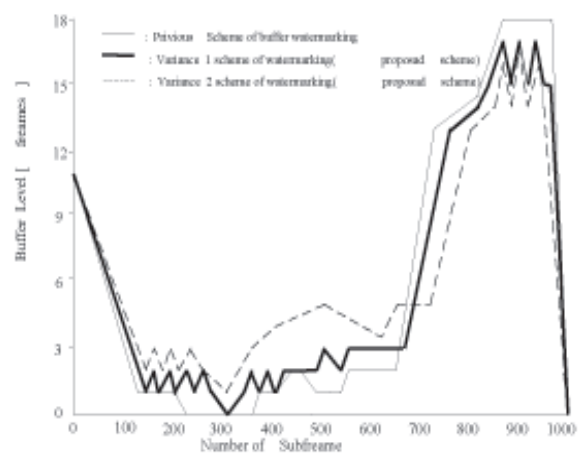

Fig. 3. Buffer Wate Marker at BS

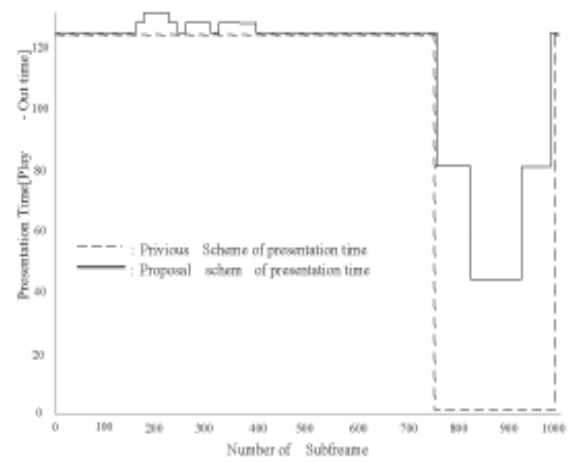

Fig. 4. Playout Time at $\mathrm{MH}$

\section{Conclusions}

In this paper we have proposed a buffering scheme of synchronization which has been brought into a core technology for supporting multimedia services. We have shown that the proposed scheme decreases the buffer size and traffic volume by separately storing multimedia data with many servers. We also prevent the buffer from experiencing the underflow or the overflow by controlling the buffer level in a presentation control scheme, and maintain the safe state of the buffer by using the evaluated arrival time as one of feedback variables in a feedback scheme. The propose scheme allows the BSs to manage the buffers and the playout policy,and provides a solution to the problems of the small memory and the limited resources.

Acknowledgements. This work was supported by the Foundation Fund of University in the Ministry of Information \& Communication 2002.

\section{References}

1. Azzedine Boukerche, Sungbum Hong and Tom Jacob, "MoSync: A Synchronization Scheme for Cellular Wireless and Mobile Multimedia System," Proceedings of the Ninth International Symposium on Modeling, Analysis and Simulation of Computer and Telecommunication Systems IEEE 2001

2. D. H. Nam and S. K. Park, "Adaptive Multimedia Stream Presentation in Mobile Computing Environment," Proceedings of IEEE TENCON, 1999. 
3. D. H. Nam, S. K. Park, "A Smooth Playback Mechanism of Media Streams in Mobile Computing Environment," ITC-CSCC'98, 1998.

4. Ernst Biersack, Werner Geyer, "Synchronization Delivery and Play-out of Distributed Stored Multimedia Streams", Multimedia Systems , V.7 N.1 , 70-90, 1999

5. M. Woo, N. Woo,N. U. Qazi, and A. Ghafoor, "A Synchronization Framework for Communication of Pre-orchestrated Multimedia Information," IEEE Network, Jan./Feb. 1994.

6. T. D. C. Little, and Arif Ghafoor, "Multimedia Synchronization Protocols for Broadband Integrated Services," IEEE Journal on selected Areas in Comm., Vol. 9, No.9, Dec. 1991.

7. W. Geyer, "Stream Synchronization in a Scalable Video Server Array," Master's thesis, Institute Eurecom, Sophia Antipolis, France, Sept., 1995. 